\title{
Correlations between choroidal abnormalities, Lisch nodules, and age in patients with neurofibromatosis type I
}

This article was published in the following Dove Press journal:

Clinical Ophthalmology

3 January 2014

Number of times this article has been viewed

\section{Shinji Makino \\ Hironobu Tampo \\ Yusuke Arai \\ Hiroto Obata}

Department of Ophthalmology, Jichi Medical University, Shimotsuke, Tochigi, Japan
Correspondence: Shinji Makino Department of Ophthalmology, Jichi Medical University, Shimotsuke, 33I I-I

Yakushiji, Tochigi, 329-0498, Japan

Tel +8 I 285587382

Fax +8I 285448365

Email makichan@jichi.ac.jp
Abstract: To evaluate correlations between choroidal abnormalities, Lisch nodules, and age in patients with neurofibromatosis type 1 (NF1), we examined ten cases with NF1 using nearinfrared reflectance imaging. Patients ranged in age from 4 to 39 years. The angle used for near-infrared reflectance imaging was $55^{\circ}$. We counted the total number of choroidal abnormalities in an area within a $55^{\circ}$ angle centered on the fovea and the total number of Lisch nodules on the iris by slit-lamp examination. No positive correlation was found between the number of Lisch nodules and patient age (Spearman's rank correlation coefficient $\rho=0.117, P=0.7414$ ). Choroidal abnormalities tended to increase with age $(\rho=0.6150)$, but this difference was not statistically significant $(P=0.0650)$. A positive correlation was found between the number of choroidal abnormalities and Lisch nodules ( $\rho=0.783, P=0.0267$ ). In conclusion, choroidal abnormalities tend to increase with patient age and are correlated with the number of Lisch nodules.

Keywords: neurofibromatosis type 1, near-infrared reflectance, choroidal abnormality, Lisch nodule, age

\section{Introduction}

Neurofibromatosis type 1 (NF1), an autosomal dominant disorder with a high mutation rate, is considered a neurocristopathy characterized by pathologic hamartomatous proliferation of neural crest-derived tissues. A minimum of two of the following criteria are required for diagnosis: six or more café-au-lait spots, two or more cutaneous neurofibromas, one or more plexiform neurofibromas, axillary or groin freckling, optic glioma, two or more Lisch nodules, distinctive bony lesions, and a first-degree relative with NF1. ${ }^{1}$ Lisch nodules in particular are frequently observed, and are a recognized feature of the disorder. However, retinal and choroidal lesions are generally considered unusual in eyes with this disease. In 2000, Yasunari et al ${ }^{2}$ suggested that choroidal abnormalities were easily detectable by infrared light examination with a scanning laser ophthalmoscope in $100 \%$ of their NF1 patients. Recently, the cutoff value for choroidal nodules detected by near-infrared reflectance was reported to be 1.5. ${ }^{3}$ Therefore, the choroid is one of the structures of the eye that is most commonly affected by NF1, and near-infrared reflectance is typically used to detect choroidal nodules in NF1 patients. ${ }^{2-6}$ To our knowledge, there have been few reports of correlations between choroidal abnormalities, Lisch nodules, and patient age. ${ }^{3,4}$ Herein, we examined ten cases of NF1 using near-infrared reflectance imaging to evaluate the correlations between number of choroidal abnormalities, Lisch nodules, and patient age. 


\section{Materials and methods}

We examined the fundus of 20 eyes of ten NF1 patients who underwent retinal imaging between May 2012 and June 2013. The patients ranged in age from 4 to 39 years. In all cases, the diagnosis of NF1 was made on the basis of the presence of several café-au-lait spots and cutaneous neurofibromas. We examined choroidal abnormalities by near-infrared reflectance (Heidelberg Retina Angiograph 2 [HRA 2], Heidelberg Engineering, Heidelberg, Germany) under mydriasis. The same operator (HT) used HRA 2 to provide $55^{\circ}$ angle fields of view. One of the authors (SM) counted the total number of choroidal abnormalities in an area within a $55^{\circ}$ angle centered on the fovea and of Lisch nodules on the iris by slit-lamp biomicroscopic examination under masking to other patient characteristics. Correlations between these choroidal abnormalities, Lisch nodules, and patient age were then analyzed. Because it was not possible to obtain an accurate count of the number of Lisch nodules in some pediatric patients, the number of nodules was determined using the following scale: I ( $\leq 1)$, II (2-4), and III ( $\geq 5)$. Differences in the number of choroidal abnormalities and Lisch nodules between the right and left eyes were analyzed by the Wilcoxon signed-rank test. Correlations between patient age, number of choroidal abnormalities, and Lisch nodules were calculated using the Spearman's rank correlation coefficient. $P$-values $<0.05$ were considered to be statistically significant. Statistical analyses were performed using Statview (version 4.5, Abacus Concepts, Berkeley, CA, USA). Three of the patients also had other clinically significant disorders, including optic glioma, astrocytic hamartoma, and congenital retinal macrovessels. ${ }^{7}$

\section{Results}

The characteristics of the ten patients are shown in Table 1. As an example, Figure 1 (case 4) shows that at least five Lisch nodules, which were classified as scale III, and 14 bright,

Table I Patient demographics and characteristics

\begin{tabular}{llll}
\hline Patient & Age & $\begin{array}{l}\text { Lisch nodule } \\
\text { R/L }\end{array}$ & $\begin{array}{l}\text { Choroidal lesion } \\
\text { R/L }\end{array}$ \\
\hline 1 & 4 & II/II & $2 / 4$ \\
2 & 6 & III/III & $3 / 8$ \\
3 & 9 & I/I & $0 / 0$ \\
4 & 9 & III/III & $7 / / 4$ \\
5 & 21 & IIIII & $1 / I$ \\
6 & 23 & III/III & $15 / 13$ \\
7 & 24 & II/II & $7 / 9$ \\
8 & 35 & III/III & $38 / 32$ \\
9 & 36 & NA & $26 / 35$ \\
10 & 39 & I/II & $8 / 10$ \\
\hline
\end{tabular}

Abbreviations: R, right; L, left; NA, not available.

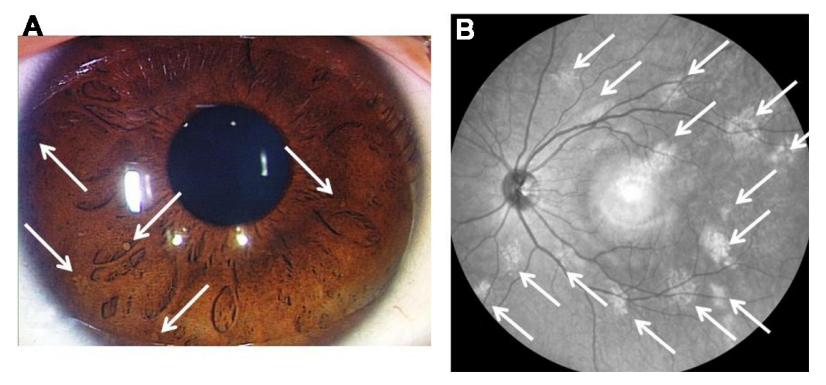

Figure I Lisch nodules and near-infrared reflectance image (case 4). At least five Lisch nodules were detected, and were classified as scale III (A). Note that 14 bright, patchy lesions were detected by near-infrared reflectance (B). The hyper-reflective point at the center of the image is an optical artifact.

patchy lesions were detected by near-infrared reflectance. There was no difference in the scale of Lisch nodules between the right and left eyes $(P=0.1025$, Wilcoxon signed-rank test). There was also no difference in the number of choroidal abnormalities between the right and left eyes $(P=0.1780)$. Therefore, we used the eye with the highest number of abnormalities in following analyses. No positive correlation was found between the scale of Lisch nodules and patient age (Spearman's rank correlation coefficient $\rho=0.117, P=0.7414$, Figure 2). Choroidal abnormalities tended to increase with patient age $(\rho=0.6150)$, but this difference was not statistically significant $(P=0.0650$, Figure 3$)$. A positive correlation was found between the number of choroidal abnormalities and the scale of Lisch nodules ( $\rho=0.783, P=0.0267$, Figure 4).

\section{Discussion}

The numbers of neurofibromas and Lisch nodules is known to increase with age. ${ }^{8,9}$ Huson et $\mathrm{al}^{9}$ reported that Lisch nodules were present in $95 \%$ of their patients $(61 / 64)$ and were bilateral in $93 \%(57 / 61)$. Among the patients aged $<16$ years $(\mathrm{n}=24)$, three had no Lisch nodules $(13 \%)$ and nine (38\%) had fewer than ten nodules per eye, whereas all patients $\geq 16$ years $(\mathrm{n}=40)$ had Lisch nodules and only

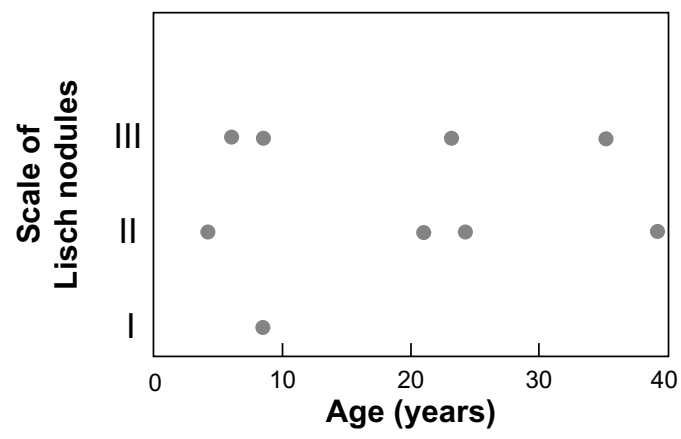

Figure 2 Correlations between scale of Lisch nodules and patient age $(\rho=0.117$, $P=0.7414)$. 


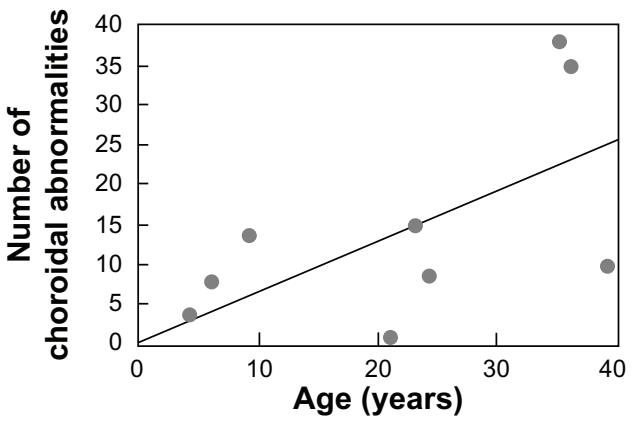

Figure 3 Correlations between number of choroidal abnormalities and patient age $(\rho=0.615, P=0.0650)$.

six had fewer than ten per eye (15\%). There was a highly significant correlation between age and number of nodules per eye. Viola et $\mathrm{al}^{3}$ reported that Lisch nodules were detected by slit-lamp examination in 68 (72\%) of $95 \mathrm{NF} 1$ subjects and in nine (43\%) of 21 pediatric NF1 subjects. In contrast, there was no significant correlation between the scale of Lisch nodules and age in this study.

To our knowledge, few reports have evaluated correlations among abnormalities, Lisch nodules, and patient age. ${ }^{3,4}$ Because choroidal abnormalities are found in all regions of the fundus, but are more frequent at the posterior pole, ${ }^{3,4} \mathrm{we}$ counted the total number of choroidal abnormalities in an area within a $55^{\circ}$ angle centered on the fovea. Nakakura et $\mathrm{al}^{4}$ found that choroidal abnormalities specific to NF1 increased significantly with patient age, and there was a positive correlation. Viola et $\mathrm{al}^{3}$ reported that bright, patchy choroidal nodules were detected by near-infrared reflectance in 79 (82\%) patients with NF1, including 15 pediatric cases (71\%). There was a statistically significant correlation between patient age and number of fundus areas involved. Viola et a ${ }^{3}$ also reported that 20 of 27 patients without Lisch nodules had choroidal nodules detected by near-infrared reflectance, and eight of 16 patients without choroidal nodules had Lisch

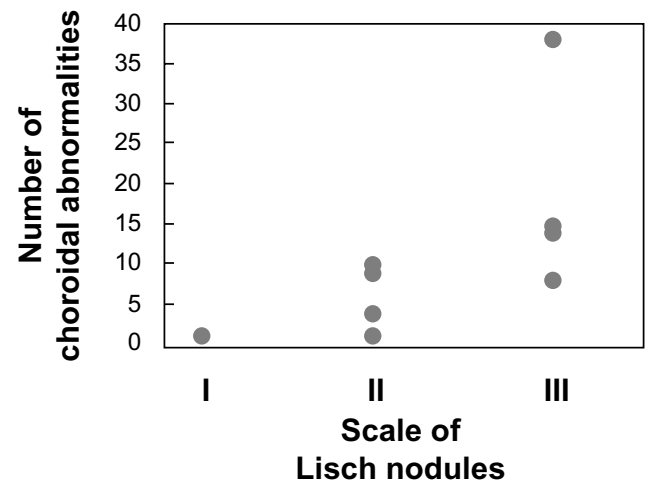

Figure 4 Correlations between number of choroidal abnormalities and scale of Lisch nodules $(\rho=0.783, P=0.0267)$. nodules. There was no significant correlation between the number of Lisch nodules and the number of choroidal nodules. In contrast, choroidal abnormalities tended to increase with age, but the difference was not statistically significant in this study. Further, a positive correlation was found between number of choroidal abnormalities and scale of the Lisch nodules.

Our study is limited by its small sample size, so we cannot reach any definitive conclusions regarding the lack of a significant correlation between number of choroidal abnormalities and patient age, or regarding the observation that choroidal abnormalities tended to increase with age. However, some of our pediatric cases undoubtedly had several choroidal abnormalities. Given that choroidal abnormalities can be easily and noninvasively observed by near-infrared reflectance and without glare compared with slit-lamp biomicroscopic examination, we consider that near-infrared reflectance examination may be useful for confirmation of the diagnosis in pediatric cases. Finally, our study was not based on longitudinal follow-up, so future studies should determine whether the number of choroidal abnormalities increases during long-term follow-up.

\section{Disclosure}

The authors report no conflicts of interest in relation to this paper.

\section{References}

1. Gutmann DH, Aylsworth A, Carey JC, et al. The diagnostic evaluation and multidisciplinary management of neurofibromatosis 1 and neurofibromatosis 2. JAMA. 1997;278:51-57.

2. Yasunari T, Shiraki K, Hattori H, Miki T. Frequency of choroidal abnormalities in neurofibromatosis type 1. Lancet. 2000;356: 988-992.

3. Viola F, Villani E, Natacci F, et al. Choroidal abnormalities detected by near-infrared reflectance imaging as a new diagnostic criterion for neurofibromatosis 1. Ophthalmology. 2012;119:369-375.

4. Nakakura S, Shiraki K, Yasunari T, Hayashi Y, Ataka S, Kohno T. Quantification and anatomic distribution of choroidal abnormalities in patients with type 1 neurofibromatosis. Graefes Arch Clin Exp Ophthalmol. 2005;243:980-984.

5. Ueda-Consolvo T, Miyakoshi A, Ozaki H, Houki S, Hayashi A. Near-infrared fundus autofluorescence-visualized melanin in the choroidal abnormalities of neurofibromatosis type 1. Clin Ophthalmol. 2012;6:1191-1194.

6. Makino S, Tampo H. Optical coherence tomography imaging of choroidal abnormalities in neurofibromatosis type 1. Case Rep Ophthalmol Med. 2013;2013:292981.

7. Makino S, Endoh K, Tampo H. Retinal microvascular abnormalities in neurofibromatosis type 1 associated with congenital retinal macrovessels. Case Rep Ophthalmol Med. 2013;2013:604191.

8. Huson SM, Harper PS, Compston DA. von Recklinghausen neurofibromatosis. A clinical and population study in south-east Wales. Brain. 1988;111:1355-1381.

9. Huson S, Jones D, Beck L. Ophthalmic manifestations of neurofibromatosis. Br J Ophthalmol. 1987;71:235-238. 


\section{Publish your work in this journal}

Clinical Ophthalmology is an international, peer-reviewed journal covering all subspecialties within ophthalmology. Key topics include: Optometry; Visual science; Pharmacology and drug therapy in eye diseases; Basic Sciences; Primary and Secondary eye care; Patient Safety and Quality of Care Improvements. This journal is indexed on
PubMed Central and CAS, and is the official journal of The Society of Clinical Ophthalmology (SCO). The manuscript management system is completely online and includes a very quick and fair peer-review system, which is all easy to use. Visit http://www.dovepress.com/ testimonials.php to read real quotes from published authors. 